\title{
Development of Mass Spectrometric Methods for Detecting Arsenic-Glutathione Complexes
}

\author{
Katerina Kanaki and Spiros A. Pergantis \\ Environmental Chemical Processes Laboratory, Department of Chemistry, University of Crete, Crete, Greece
}

It has been suggested recently that arsenic-glutathione (As-GSH) complexes play an important role in the methylation of arsenic. The present study describes the development of high-performance liquid chromatography (HPLC)-electrospray tandem mass spectrometry (ES-MS/MS), operated in the selected reaction monitoring (SRM) mode, and HPLCinductively coupled plasma mass spectrometry (ICP-MS) methods suitable for the sensitive and selective identification of four As-GSH complexes. Method optimization was carried out using a series of synthetically prepared standards, i.e., three As-GSH species containing trivalent arsenic: tri(glutamyl-cysteinyl-glycinyl)trithio-arsenite (ATG), di(glutamyl-cysteinyl-glycinyl)methyldithio-arsonite (MADG), and (ã-glutamyl-cysteinyl-glycinyl) dimethyl-thio-arsinite (DMAG), as well as one As-GSH species containing pentavalent As: dimethylthioarsinic acid-glutathione $\left(\mathrm{DMTA}^{\mathrm{v}}-\mathrm{GSH}\right)$. The collision induced dissociation behavior of these compounds was investigated in detail to identify optimum SRM transitions for each complex. Both methods were based on reversed-phase chromatography using gradient elution with methanol, formic acid, and water as solvents. The amount of methanol that was used with this HPLC method (up to $12 \% \mathrm{vol} / \mathrm{vol}$ ) was compatible with ICP-MS, without the need of a specially adapted interface. Subsequently, these analytical methods were applied to carry out a preliminary investigation about the role of As-GSH complexes in the methylation of arsenite by methylcobalamin $\left(\mathrm{CH}_{3} \mathrm{~B}_{12}\right)$ in the presence of glutathione (GSH). For the first time, the complexes ATG, MADG, and trace amounts of DMAG were detected as products of this reaction. (J Am Soc Mass Spectrom 2008, 19, 1559-1567) (C 2008 American Society for Mass Spectrometry

W orldwide, several millions of people are now affected by elevated arsenic levels in their drinking water [1]. The predominant forms of arsenic in drinking water are arsenite and arsenate (iAs ${ }^{I I I}$ and $i A s^{V}$, respectively), the inorganic species which are regarded as human carcinogens $[2,3]$. Inorganic arsenic compounds are transformed in the body by repetitive reduction and oxidative methylation reactions, leading to the formation of organoarsenic species, the major of which are monomethylarsonic acid $\left(\mathrm{MMA}^{\mathrm{v}}\right)$ and dimethylarsinic acid $\left(\mathrm{DMA}^{\mathrm{V}}\right)[3,4]$.

The precise pathway through which inorganic arsenic is metabolized in the organism is not yet known in sufficient detail. The generally accepted methylation mechanism involves the initial conversion of pentavalent arsenic species $\mathrm{iAs}^{\mathrm{V}}$ to trivalent species iAs ${ }^{\mathrm{III}}$ through a two-electron reduction, while oxidative addition of a methyl group follows $[4,5]$. Glutathione (GSH) can act as a reducing agent and S-adenosylmethionine (SAM) as a methyl donor. The major site for the methylation seems to be the liver. However, Hayakawa et al. recently proposed

Address reprint requests to Dr. S. A. Pergantis, Environmental Chemical Processes Laboratory, Department of Chemistry, University of Crete, Voutes, Heraklion 71003, Crete, Greece. E-mail: spergantis@chemistry. uoc.gr an alternative mechanism for the methylation of inorganic arsenic in biological systems [6]. According to this methylation mechanism, complexes of arsenic with glutathione (As-GSH) are believed to play an essential role in the metabolism and transport of inorganic arsenic within an organism.

Arsenic has a strong affinity for sulfur as indicated by the numerous As-S compounds containing pentavalent arsenic that have been identified in biological samples recently [7-10]. However, most of these As-S species comprise small molecules that do not contain amino acids. So far, only a few arsenic-peptide species, formed via As-S bonds, have been identified in vivo, such as a series of arsenic phytochelatin complexes in plants [11] and arsenic glutathione complexes in bile and urine [12-15], all of which contain trivalent arsenic. The first study which showed that pentavalent arsenic can also bind to biomolecules was published in 2007 by Feldmann et al. [16]. This study demonstrated the formation of the pentavalent dimethylthioarsinic acidglutathione complex (DMTA $\left.{ }^{\mathrm{V}}-\mathrm{GSH}\right)$ in cabbage (Brassica oleracea) when exposed to $\mathrm{DMA}^{\mathrm{V}}$. This finding not only changes the view of how arsenic may interact with other sulfur-rich biomolecules, but also highlights the fact that As-S interactions may have a key role in the reactivity of arsenic intermediates and the metabolic pathway of arsenic in an organism. 
From previous studies, it is known that As-GSH conjugates are not stable under the conventional chromatographic methods used in arsenic speciation analysis involving As species having As-C and As-O bonds. More specifically, it has been reported that these AsGSH complexes decompose on the PRPX-100 anion exchange HPLC column, when carbonate or formic acid buffers are used [17]. This is most likely the reason that has made their detection in biological tissues so problematic. On the other hand, the fact that no precautions are usually taken during extraction procedures to prevent their oxidation may have also contributed to their late detection.

The first attempts to detect and characterize trivalent As-GSH complexes were based on nuclear magnetic resonance (NMR) spectroscopy, by observing the chemical shifts of the proton $\left({ }^{1} \mathrm{H}\right)$ and the carbon$13\left({ }^{13} \mathrm{C}\right)$ nuclei $[18,19]$. This technique led to the identification of the synthetically derived ATG [tri(glutamyl-cysteinyl-glycinyl)trithio-arsenite], MADG [di(glutamyl-cysteinyl-glycinyl)methyl-dithio-arsonite], and DMAG [(ã-glutamyl-cysteinyl-glycinyl)dimethyl-thioarsinite] complexes, which were derived from the reaction of $\mathrm{iAs}^{\mathrm{III}}, \mathrm{MMA}^{\mathrm{V}}$, and $\mathrm{DMA}^{\mathrm{V}}$, respectively, with GSH. Subsequently, thin-layer chromatography (TLC) using radiolabeled arsenicals with the isotope ${ }^{73} \mathrm{As}$ was applied for the identification of the specific complexes $[20,21]$. However, both of these techniques do not offer the sensitivity and selectivity that is demanded for the detection of the species at the low $\mu \mathrm{g} / \mathrm{L}$ level that they may be found in biological samples. In addition, "open column liquid chromatographic" techniques do not ensure the stability of the analyzed compounds, while the use of radioactive isotopes, such as ${ }^{73} \mathrm{As}$, is technically quite complicated and generally not desirable. For these reasons, researchers attempted to improve the existing analytical capabilities by using modern state-of-the-art analytical techniques, such as highperformance liquid chromatography (HPLC)-inductively coupled plasma mass spectrometry (ICP-MS) and HPLC-electrospray tandem mass spectrometry (ESMS/MS). Kala et al., using reversed-phase chromatography in combination with ICP-MS and ES-MS (in selected ion monitoring mode), managed to identify the complexes ATG and MADG in rat's bile after the administration of sodium arsenite [12]. However, the separation under these conditions was not sufficient, which is a significant disadvantage during the analysis with HPLC-ICP-MS. In subsequent studies, the chromatography was improved, giving well-defined and fully separated peaks for the three As-GSH complexes (ATG, MADG, and DMAG), during analysis with both ICP-MS and ES-MS [17]. Although the ES-MS technique has been used for the identification of trivalent As-GSH complexes in standard solutions as well as in biological samples, by facilitating the observation of $m / z$ values corresponding to precursor molecular ions, only one study up to now uses tandem spectrometry (MS/MS) to obtain additional structural information regarding the compounds of interest [22]. Nevertheless, since this study did not focus on arsenic, only a few fragment ions were presented. With respect to the pentavalent As-GSH complex (DMTA $\left.{ }^{\mathrm{V}}-\mathrm{GSH}\right)$, its identification has been carried out using reversed-phase HPLC coupled simultaneously to ES-MS/MS and ICP-MS detectors [16].

In the present study, emphasis was given to the development of a HPLC-ES-MS/MS method in selected reaction monitoring (SRM) mode suitable for the simultaneous detection and characterization of the three trivalent As-GSH complexes, i.e., ATG, MADG, and DMAG, as well as the one pentavalent As-GSH complex (DMTA $\left.{ }^{\mathrm{V}}-\mathrm{GSH}\right)$. SRM was chosen as a very sensitive and selective approach that allows for interferencefree identification of analytes at the low $\mu \mathrm{g} / \mathrm{L}$ level even in crude biological extracts containing high levels of matrix. The presence of the four As-GSH species was further confirmed using HPLC-inductively coupled plasma mass spectrometry (ICP-MS). It is envisioned that because of their sensitivity and selectivity, this combination of techniques may potentially be used to investigate the presence of As-GSH species in crude biological samples. Both methods were based on reversed-phase chromatography with gradient elution, which allows for the efficient elution of the complexes from the column and their sufficient separation.

The HPLC-ICP-MS and HPLC-ES-MS/MS methods developed in this study were further used to investigate the formation of As-GSH species in arsenic methylation reactions, in particular for investigating the nonenzymatic methylation of arsenite by methylcobalamin in the presence of glutathione. This reaction has previously been studied, although the presence of As-GSH conjugates amongst the reaction products has not been reported until now [23, 24].

\section{Experimental}

\section{Chemicals}

Sodium arsenite $\left(\mathrm{NaAsO}_{2}\right)$ was purchased from $\mathrm{BDH}$ Ltd., Poole, United Kingdom. Monosodium acid methane arsonate sesquihydrate (purity 99\%) was purchased from Supelco (Bellefonte, PA). Cacodylic acid $\left(\mathrm{C}_{2} \mathrm{H}_{7} \mathrm{AsO}_{2}\right.$, puriss. $\left.\geq 99 \%\right)$, L-glutathione reduced $\left(\mathrm{C}_{10} \mathrm{H}_{17} \mathrm{~N}_{3} \mathrm{O}_{6} \mathrm{~S}\right.$, puriss. $\left.\geq 97 \%\right)$, tris (hydroxymethyl) aminomethane hydrochloride $\left(\mathrm{C}_{4} \mathrm{H}_{11} \mathrm{NO}_{3} \cdot \mathrm{HCl}\right.$, ultra for molecular biology), formic acid $\left(\mathrm{CH}_{2} \mathrm{O}_{2}\right.$, puriss. p.a. ACS; $88 \%-91 \%\{\mathrm{~T}\})$, and ammonium hydroxide solution $\left(\mathrm{NH}_{5} \mathrm{O}\right.$, puriss., p.a.) were purchased from Fluka Chemika, Buchs, Switzerland. Methylcobalamin $\left(\mathrm{C}_{63} \mathrm{H}_{91} \mathrm{CoN}_{13} \mathrm{O}_{14} \mathrm{P}\right)$ and methanol $\left(\mathrm{CH}_{3} \mathrm{OH}\right.$, for gradient elution ACS) were purchased from Sigma (Buchs, Switzerland). Hydrochloric acid ( $\mathrm{HCl}, 37 \%$, puriss., p.a.) and iron(II) sulfide sticks (Fe $\left.{ }^{\mathrm{II}} \mathrm{S}\right)$ were obtained from Riedel-de Haen, Seelze, Germany. 


\section{Synthesis of As-GSH Complexes}

The synthesis of ATG, MADG, DMAG, and DMTA ${ }^{\mathrm{V}}$ GSH complexes was based on previously published procedures $[12,16,18]$. With respect to the trivalent As-GSH complexes (ATG, MADG, and DMAG) the reaction solution was left at room temperature overnight under an argon atmosphere, with continuous stirring.

More specifically, ATG was prepared by dissolving sodium arsenite $(0.037 \mathrm{~g} ; 0.28 \mathrm{mmol})$ and GSH reduced $(0.26 \mathrm{~g} ; 0.85 \mathrm{mmol})$ in $2.5 \mathrm{~mL}$ of degassed deionized water. At the end of the reaction, the product was precipitated with $10 \mathrm{~mL}$ methanol. The precipitate was centrifuged, and following the removal of the supernatant, the residue was dried at room temperature.

Similarly, MADG was prepared by dissolving monosodium acid methane arsonate $(0.059 \mathrm{~g}, 0.36$ mmol) and GSH $(0.5 \mathrm{~g}, 1.63 \mathrm{mmol})$ in $2 \mathrm{~mL}$ of degassed deionized water. At the end of the reaction, the product was precipitated with $10 \mathrm{~mL}$ ethanol. The precipitate was centrifuged, and following the removal of the supernatant, the residue was dried at room temperature.

For DMAG preparation, cacodylic acid $(0.035 \mathrm{~g} ; 0.25$ $\mathrm{mmol})$ and GSH $(0.23 \mathrm{~g} ; 0.75 \mathrm{mmol})$ were dissolved in $2.5 \mathrm{~mL}$ of degassed deionized water. At the end of the reaction, the product was precipitated with $10 \mathrm{~mL}$ of methanol. The precipitate was centrifuged, and following the removal of the supernatant the residue was dried at room temperature.

Following the synthesis of the trivalent As-GSH complexes, as described previously, the dried solid corresponding to each complex was reconstituted in 1 $\mathrm{mL}$ of $0.1 \%$ formic acid solution and then diluted further before analysis with HPLC-ES-MS/MS and HPLC-ICP-MS.

With respect to the synthesis of the pentavalent As containing compound DMTA ${ }^{\mathrm{V}}-\mathrm{GSH}$, initially a solution containing dimethylthioarsinic acid $\left(\mathrm{DMTA}^{\mathrm{V}}\right)$ was prepared by treating a $\mathrm{DMA}^{\mathrm{V}}$ containing solution (10 ppm) with $\mathrm{H}_{2} \mathrm{~S}$ (which resulted from the reaction of $\mathrm{Fe}^{\mathrm{II} S}$ with hydrochloric acid). Then, equal volumes of a GSH containing solution $(214 \mathrm{mM})$ and a DMTA ${ }^{\mathrm{V}}$ containing solution were mixed and left to react for 45 min before analysis using HPLC-ICP-MS and HPLCES-MS/MS.

\section{Arsenite Methylation Reaction}

To investigate the methylation of arsenite by methylcobalamin $\left(\mathrm{CH}_{3} \mathrm{~B}_{12}\right)$ in the presence of GSH, a solution containing $0.06 \mathrm{M}$ tris buffer ( $\mathrm{pH} 7.8$ ), $45 \mathrm{mM} \mathrm{GSH}$ reduced, and $16 \mathrm{ppm}$ sodium arsenite was prepared. The mixture was left to stand under an argon atmosphere for $45 \mathrm{~min}$ at $37^{\circ} \mathrm{C}$ before adding the methylcobalamin solution (giving a final concentration of 0.17 $\mathrm{mM}$ methylcobalamin in the reaction solution). Micro- liter aliquots were taken from the reaction mixture at various time intervals, and analyzed immediately with and without dilution by using HPLC-ICP-MS and HPLC-ES-MS/MS.

\section{Instrumentation}

For the HPLC-ES-MS/MS method, a triple quadrupole TSQ Quantum mass spectrometer (Thermo Finnigan, San Jose, CA) was used with an electrospray ion source in the positive ion mode. The optimum tune parameters were the following: electrospray voltage $4.1 \mathrm{kV}$, sheath gas pressure 45 arbitrary units, auxiliary gas pressure 25 arbitrary units, capillary temperature $300{ }^{\circ} \mathrm{C}$, source collision-induced dissociation (CID) $0 \mathrm{~V}$.

For the HPLC-ICP-MS method, an X-series ICP mass spectrometer was used (Thermo Fischer Scientific, Winsford, United Kingdom) to monitor ions at $\mathrm{m} / \mathrm{z} 75$ (As) and $m / z 77\left(\mathrm{ArCl}^{+}\right)$. The optimum tune parameters used were the following: forward power $1400 \mathrm{~W}$, nebulizer gas flow rate $1.0 \mathrm{~mL} / \mathrm{min}$, cooling gas flow rate $13.5 \mathrm{~L} / \mathrm{min}$, auxiliary gas flow rate $1.0 \mathrm{~L} / \mathrm{min}$, lens 1 voltage $-0.6 \mathrm{~V}$, extraction lens voltage $-196 \mathrm{~V}$.

For both methods, reversed-phase chromatography with a BDS hypersil C18 chromatographic column (150 $\mathrm{mm} \times 2.1 \mathrm{~mm}, 5-\mu \mathrm{m}$ particle size, Thermo) and a BDS Hypersil C18 precolumn (Thermo) was applied. After trying several different HPLC gradient modes using various amounts of methanol and formic acid in the mobile phase, a set of optimum mobile phase conditions was reached, and gradient elution was performed using a combination of $0.05 \% \mathrm{vol} / \mathrm{vol}$ concentrated $(d=$ $1.20 \mathrm{~g} / \mathrm{mL}$ ) formic acid in $2 \% \mathrm{vol} / \mathrm{vol}$ aqueous methanol at $\mathrm{pH} \sim 3$ (Eluent A) and 100\% vol/vol methanol (Eluent B). The gradient was performed using an LC20-AD Prominence solvent delivery system (Shimadzu Corp., Kyoto, Japan). The detailed gradient program was as follows: $0-10 \mathrm{~min} 100 \%$ Eluent $\mathrm{A}$ and $0 \%$ Eluent $B ; 10-11$ min changed to $90 \%$ Eluent $A$ and $10 \%$ Eluent B; $11-30$ min constant at $90 \%$ Eluent $A$ and $10 \%$ Eluent B; $31-40$ min changed to $100 \%$ Eluent A.

The HPLC flow rate both for HPLC-ES-MS/MS and HPLC-ICP-MS methods was $0.2 \mathrm{~mL} / \mathrm{min}$. With respect to the ES-MS/MS, $100 \%$ of the eluent was directed to the ES source, while in the case of ICP-MS, there was a post column make-up flow of dilute nitric acid $(2 \%$ $\mathrm{vol} / \mathrm{vol})$ delivered via a peristaltic pump $(\sim 0.9 \mathrm{~mL} /$ $\mathrm{min}$ ). The role of the make-up flow was first to further dilute the organic content of the eluent (methanol) and then to increase the flow introduced to the ICP to have efficient nebulization.

With this optimized method, the complete separation of all four As-GSH complexes was achieved, with sharp and well defined peaks, while retention times corresponding to the complexes were stable and identical for both standards and samples. 


\section{Results and Discussion}

\section{Development of an HPLC-ES-MS/MS Method for} the Identification of As-GSH Complexes

Product ion scan. Reversed-phase HPLC-ES-MS/MS experiments were conducted to characterize the AsGSH content of all the standard solutions prepared in the present study. For this reason, initially, product ion scanning in the positive ion mode was applied for the analysis of standard solutions containing the As-GSH complexes (ATG, MADG, DMAG, and DMTA ${ }^{\mathrm{V}}-\mathrm{GSH}$ ), $\mathrm{GSH}$, and $\mathrm{DMA}^{\mathrm{v}}$. In each of these product ion scans, the protonated molecule corresponding to each compound was selected as the precursor ion. The expected $\mathrm{m} / \mathrm{z}$ ratios for the protonated molecules of ATG, MADG, DMAG, DMTA ${ }^{\mathrm{V}}-\mathrm{GSH}, \mathrm{GSH}$, and DMA ${ }^{\mathrm{V}}$ are $\mathrm{m} / \mathrm{z}$ 994, $703,412,444,308$, and 139, respectively. Upon collision induced dissociation (CID) of the precursor ions at various collision energies $(5,15,25,35,45$, and $60 \mathrm{eV})$, the product ion mass spectra corresponding to each of these species were recorded. Representative product ion mass spectra of the four As-GSH species are shown in Figure 1. Moreover, the mass spectra of $\mathrm{DMA}^{\mathrm{V}}$ and GSH are presented as Supplementary Figure 1, which can be found in the electronic version of this article. The
CID breakdown curves corresponding to these compounds, displaying the absolute intensities of the major product ions as a function of the collision energy, are shown in Supplementary Figure 2. The mass spectra of all the species revealed the presence of characteristic product ions. The proposed structures of the product ions corresponding to the four As-GSH species are shown in Scheme 1, while the proposed structures of the product ions corresponding to $\mathrm{DMA}^{\mathrm{v}}$ and GSH are presented in Supplementary Scheme $\mathbf{1}$.

Because of the low sensitivity observed for $\mathrm{MMA}^{\mathrm{V}}$ under positive ion electrospray ionization conditions, we were not able to obtain product ion scanning data for this compound, so previously published SRM transitions were used [25].

Figure 1a presents the tandem mass spectrum of the protonated molecule having $\mathrm{m} / \mathrm{z}$ 994, which corresponds to the protonated ATG complex. At collision energy $25 \mathrm{eV}$, apart from observing the precursor molecular ion $(\mathrm{m} / \mathrm{z} 994)$, a product ion at $\mathrm{m} / \mathrm{z} 687$ also predominates. The latter derives from the precursor ion following the elimination of a GSH molecule. Other notable product ions are those having $\mathrm{m} / \mathrm{z} 308$ (which corresponds to the protonated GSH molecule), 380 (which arises from the product ion with $\mathrm{m} / \mathrm{z} 687$ after

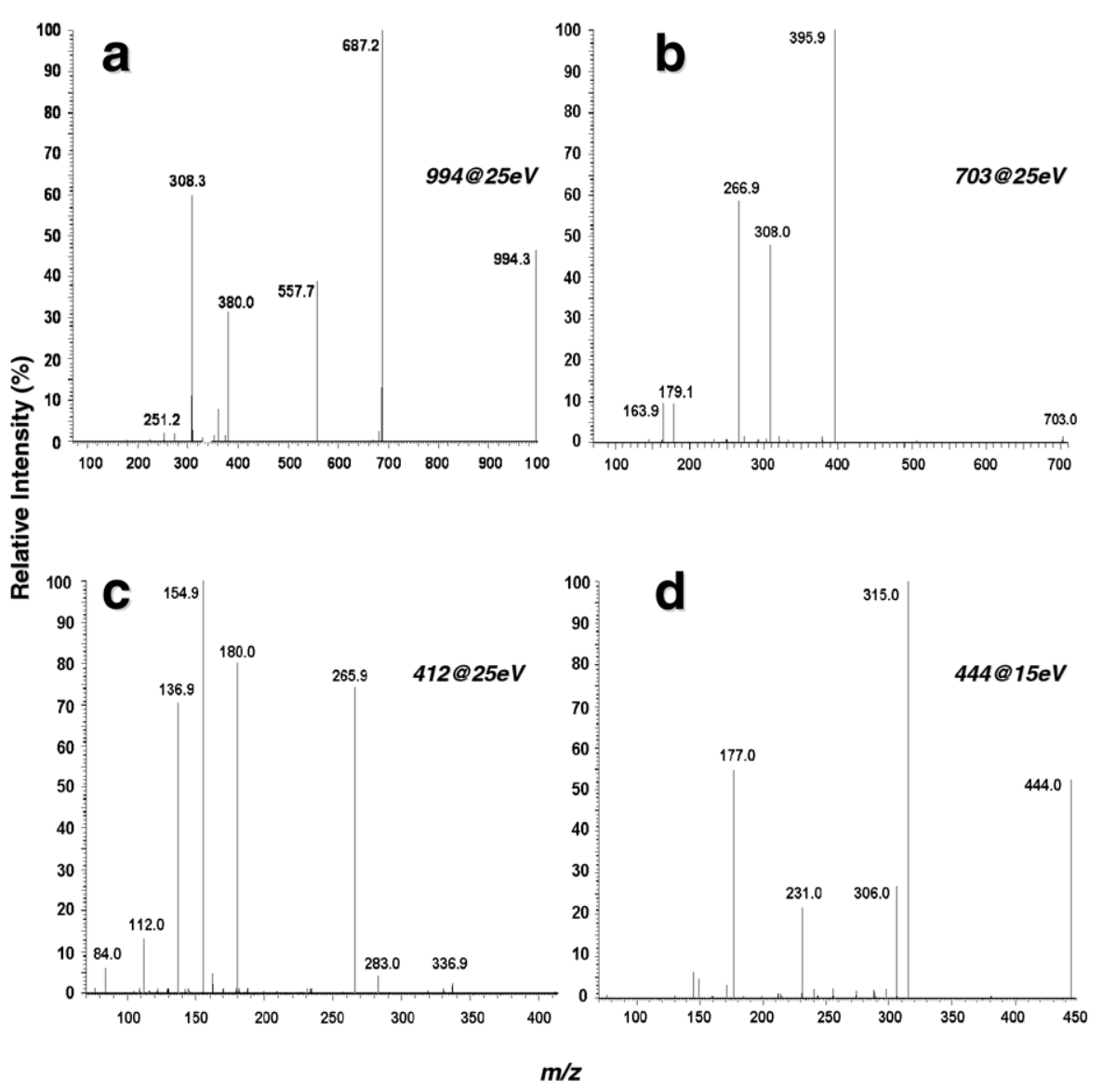

Figure 1. Product ion mass spectra of the protonated molecules of: (a) ATG, (b) MADG, (c) DMAG, and (d) DMTA ${ }^{\mathrm{v}}-\mathrm{GSH}$. 
a
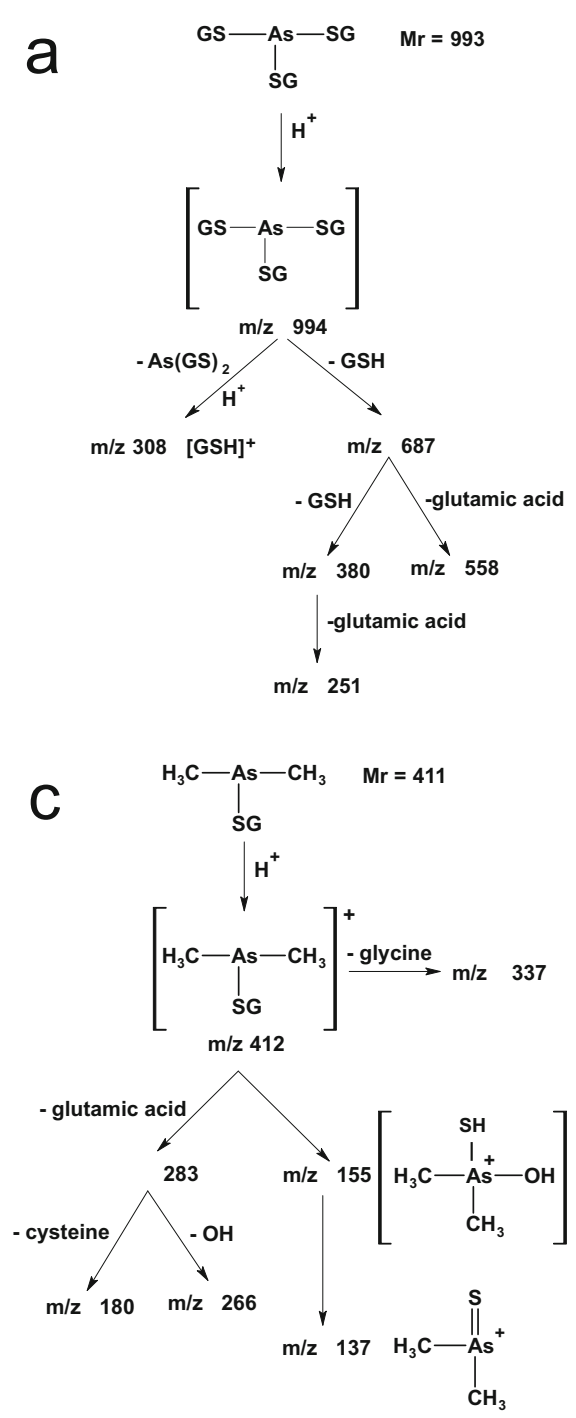

b
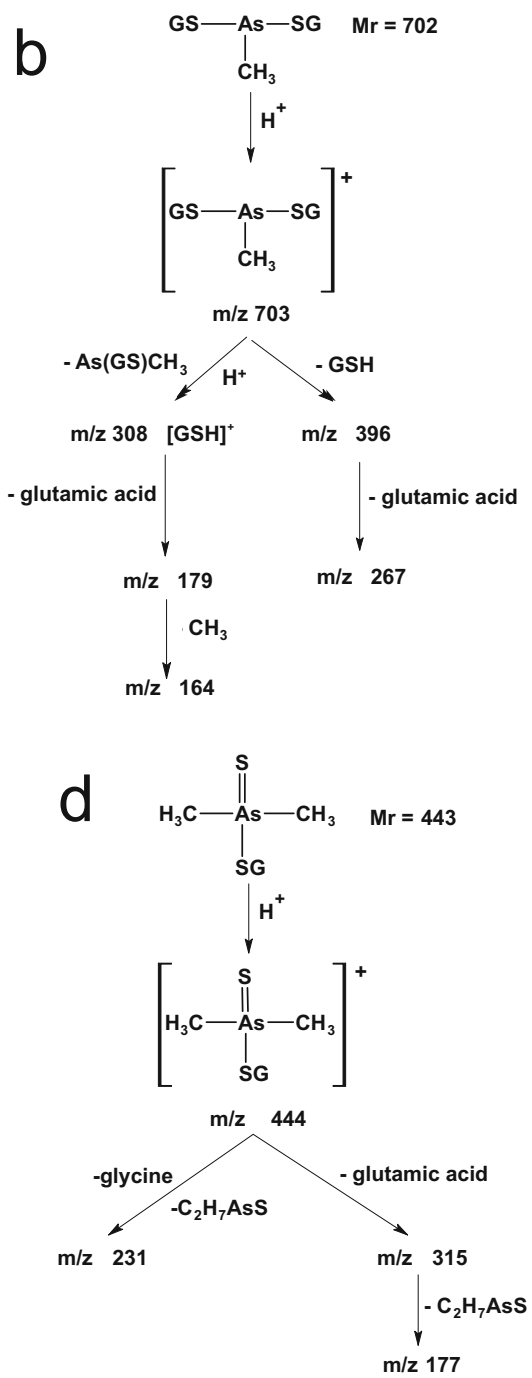

Scheme 1. Collision induced dissociation (CID) behavior of: (a) ATG, (b) MADG, (c) DMAG, and (d) DMTA ${ }^{\mathrm{V}}-\mathrm{GSH}$.

the loss of another GSH molecule), and 251 (which results from the product ion with $\mathrm{m} / \mathrm{z} 380$ after the loss of glutamic acid). The suggested CID pathways of these complexes are shown in Scheme 1a. The loss of glutamic acid is typical for the CID of GSH. Other observed product ions are the ones with $\mathrm{m} / \mathrm{z} 558$, which derive from the ions with $m / z 687$ after the loss of glutamic acid (Scheme 1a).

Figure $1 \mathrm{~b}$ presents the tandem mass spectrum of the protonated molecule with $\mathrm{m} / \mathrm{z} 703$, which corresponds to MADG. The CID behavior of this species is similar to that of ATG, which was presented previously. At a collision energy of $25 \mathrm{eV}$, the product ion with $\mathrm{m} / \mathrm{z} 396$ predominates, and is believed to derive from the precursor ion following the loss of a GSH molecule. The product ion with $\mathrm{m} / \mathrm{z} 308$, which corresponds to the protonated molecule of GSH, is also observed under these MS/MS conditions. The fragment ions with $\mathrm{m} / \mathrm{z}$ 267 and 179, resulting from the product ions with $\mathrm{m} / \mathrm{z}$
396 and 308, respectively, following the elimination of glutamic acid, were also observed (Scheme 1b). Finally, other observed product ions are those with $\mathrm{m} / \mathrm{z} 164$ as shown in Figure 1b.

The tandem mass spectrum of the protonated molecule with $m / z 412$, which corresponds to the complex DMAG, are shown in Figure 1c. At a collision energy of $25 \mathrm{eV}$, the product ions with $\mathrm{m} / \mathrm{z} 283,266,180,155$, and 137 predominate. The former product ion is believed to result from the precursor ion following the loss of glutamic acid, while its further fragmentation leads to the removal of a $-\mathrm{OH}$ group and the formation of an ion with $\mathrm{m} / \mathrm{z} 266$. The product ion with $\mathrm{m} / \mathrm{z} 337$ results from the precursor ion after the loss of a glycine molecule. Finally, the product ions with $\mathrm{m} / \mathrm{z} 180,155$, and 137 have the structures suggested in Scheme 1c.

Figure 1d presents the tandem mass spectrum of the protonated molecule with $\mathrm{m} / \mathrm{z} 444$, which corresponds to DMTA ${ }^{\mathrm{V}}-\mathrm{GSH}$. This mass spectrum revealed an 
abundant species at $m / z 315$, which corresponds to the loss of glutamic acid from the precursor ion. Other product ions observed occur at $\mathrm{m} / \mathrm{z} 231$ [which results from the precursor ion after the loss of a glycine molecule and the $\left(\mathrm{CH}_{3}\right)_{2} \mathrm{AsSH}$ moiety] and 177 [which results from the product ion with $m / z 315$, after the loss of the $\left(\mathrm{CH}_{3}\right)_{2} \mathrm{AsSH}$ moiety]. The resulting product ion mass spectra for this compound agree with the ones reported by Feldmann et al. [16]. The interpretation of these mass spectra is provided in Scheme $\mathbf{1 d}$.

Supplementary Figure 1a presents the mass spectra of the protonated molecule with $\mathrm{m} / \mathrm{z} 308$, which corresponds to GSH. These spectra revealed abundant species of $\mathrm{m} / \mathrm{z} 179$ and 233, which resulted from the precursor ion after the elimination of glutamic acid and glycine respectively. From the ion with $m / z 179$, after further fragmentation and removal of a $-\mathrm{OH}$ group, the product ion with $\mathrm{m} / \mathrm{z} 162$ resulted. Other fragment ions that are observed are the ones at $m / z 144,116$, and 76 (Supplementary Scheme 1a).

Finally, in Supplementary Figure $1 \mathrm{~b}$ the mass spectra of the protonated $\mathrm{DMA}^{\mathrm{V}}$ molecule with $\mathrm{m} / \mathrm{z} 139$ are presented. The interpretation of these spectra is described in Supplementary Scheme 1b.

Selected reaction monitoring (SRM). The breakdown curves provided as Supplementary Figure 2 were used to find the most efficient conditions for generating characteristic product ions for each arsenic species and for GSH to be used in the selected reaction monitoring (SRM) mode. The SRM approach is invaluable as it enables sensitive and selective detection, if appropriate conditions are used. The SRM conditions were optimized for each analyte by selecting the most abundant product ions with their corresponding optimum collision energies. From the CID breakdown curves obtained in this study, the two most efficient fragmentation reactions were selected for each arsenic species and for GSH (Table 1). However, for the detection of MMA only one SRM transition was used, as presented in a previous study [25].

Using the HPLC-ES-MS/MS method in the SRM mode, a standard solution containing all four As-GSH complexes (ATG, MADG, DMAG, and DMTA ${ }^{\mathrm{V}}-\mathrm{GSH}$ ),

Table 1. SRM conditions used for the detection of three As-GSH complexes, GSH, MMA ${ }^{\mathrm{V}}$, and DMA ${ }^{\mathrm{V}}$

\begin{tabular}{|c|c|c|}
\hline & \multicolumn{2}{|c|}{$\begin{array}{c}\text { Selected reactions monitored (collision } \\
\text { energy/eV) }\end{array}$} \\
\hline & SRM transition 1 & SRM transition 2 \\
\hline $\mathrm{MMA}^{\mathrm{V}}$ & $141 \rightarrow 91(30 \mathrm{eV})$ & - \\
\hline DMA $^{\mathrm{V}}$ & $139 \rightarrow 91(25 \mathrm{eV})$ & $139 \rightarrow 109(25 \mathrm{eV})$ \\
\hline GSH & $308 \rightarrow 179(15 \mathrm{eV})$ & $308 \rightarrow 162(15 \mathrm{eV})$ \\
\hline ATG & $994 \rightarrow 308(35 \mathrm{eV})$ & $994 \rightarrow 687(25 \mathrm{eV})$ \\
\hline MADG & $703 \rightarrow 308(17 \mathrm{eV})$ & $703 \rightarrow 396(25 \mathrm{eV})$ \\
\hline DMAG & $412 \rightarrow 137(35 \mathrm{eV})$ & $412 \rightarrow 155(25 \mathrm{eV})$ \\
\hline DMTA $^{\mathrm{V}}-\mathrm{GSH}$ & $444 \rightarrow 315(15 \mathrm{eV})$ & $444 \rightarrow 177(25 \mathrm{eV})$ \\
\hline
\end{tabular}

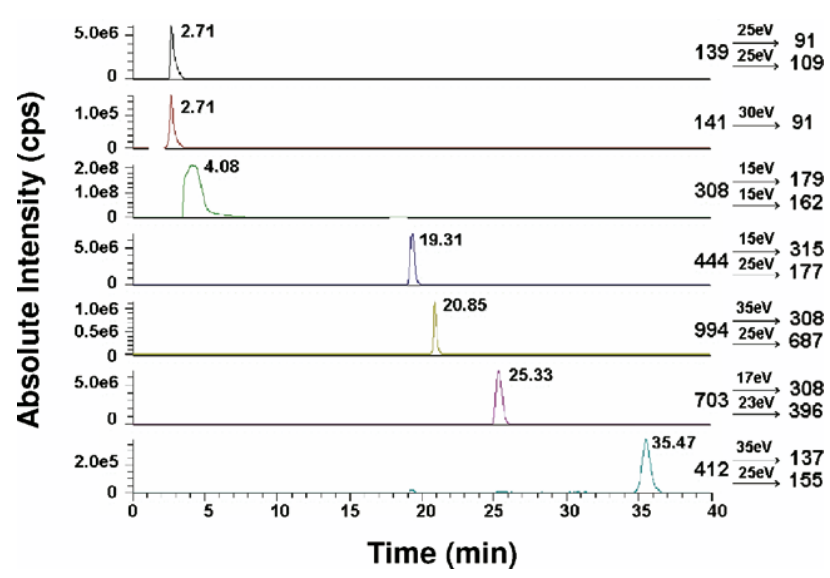

Figure 2. HPLC-ES-MS/MS-SRM chromatogram of a standard solution containing: DMA ${ }^{\mathrm{V}}, \mathrm{MMA}^{\mathrm{V}}, \mathrm{GSH}$, ATG $(35 \mu \mathrm{g} / \mathrm{mL})$, MADG $(12 \mu \mathrm{g} / \mathrm{mL})$, DMAG $(9 \mu \mathrm{g} / \mathrm{mL})$, and DMTA ${ }^{\mathrm{V}}-\mathrm{GSH}(0.22$ $\mu \mathrm{g} / \mathrm{mL})$.

along with $\mathrm{MMA}^{\mathrm{V}}, \mathrm{DMA}^{\mathrm{v}}$, and GSH, was analyzed. The resulting chromatogram (Figure 2) revealed the presence of all seven species contained in the mixture, with all four As-GSH complexes being well resolved.

Subsequently, an aliquot removed from the arsenite

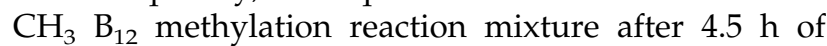
reaction was analyzed under the same HPLC-ES-MS/ MS-SRM conditions. The resulting chromatogram, shown in Figure 3, revealed the presence of ATG (20.77 min) and MADG (25.39 min) complexes, while a small peak corresponding to DMAG (35.50 $\mathrm{min})$ was also observed. In the same chromatogram, the presence of large amounts of GSH is also obvious. Peaks for the $\mathrm{DMA}^{\mathrm{V}}$ and $\mathrm{MMA}^{\mathrm{V}}$ were not observed, which may be due to the low sensitivity exhibited for these two species in positive ion mode ES-MS/MS.

Subsequently, a "blank" methylation reaction mixture (i.e., no arsenic added to the reaction mixture) was analyzed to exclude the possibility that the peaks attributed to ATG, MADG, and DMAG in the arsenite

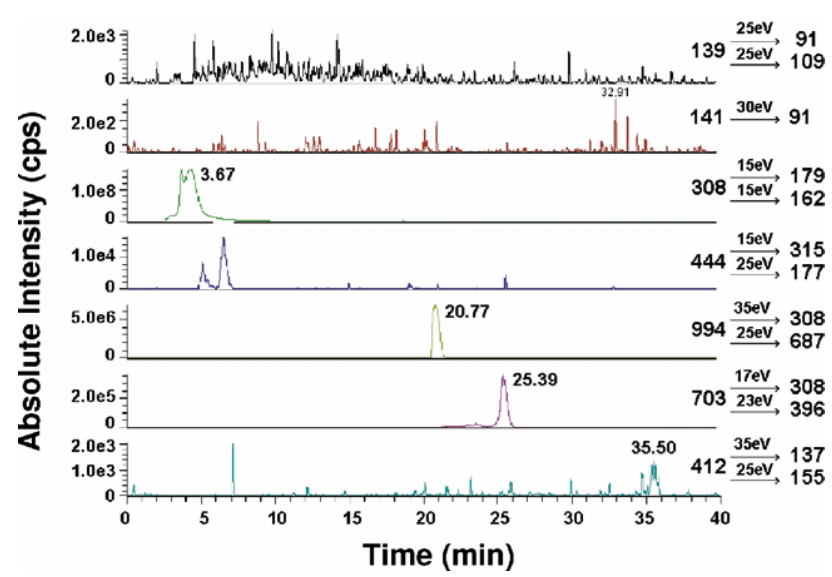

Figure 3. HPLC-ES-MS/MS-SRM chromatogram of the arsenite methylation reaction mixture after $4.5 \mathrm{~h}$ incubation at $37^{\circ} \mathrm{C}$. 
methylation mixture were artifacts. None of these peaks was in fact observed in the "blank" mixture.

A major advantage of the detection of arsenic species by HPLC-ES-MS/MS in the SRM mode is that standards are not a prerequisite. The detailed CID data reported here can be used by other researchers, without the need for standards, to detect the presence of such As-GSH complexes.

\section{Development of a HPLC-ICP-MS Method for the Identification of As-GSH Complexes}

After the development of the HPLC-ES-MS/MS method for the detection and characterization of the As-GSH conjugates, attempts were made to apply the same HPLC method with ICP-MS detection. The solvents applied with the HPLC-ES-MS/MS technique were compatible with the ICP-MS. The methanol that was used did not exceed $12 \% \mathrm{vol} / \mathrm{vol}$, an amount that was further diluted using a make-up flow before introduction into the ICP-MS nebulizer.

Initially, standard solutions of As-GSH complexes were analyzed using this approach, and the resulting chromatograms are shown in Figure 4. The observed retention times for the four As-GSH complexes are similar to the ones observed during the analysis of the standard solutions with HPLC-ES-MS/MS. Some variation may be attributable to the different HPLC instrumentation used in each case. The ATG, MADG, DMAG, and DMTA ${ }^{\mathrm{V}}-\mathrm{GSH}$ species correspond to the peaks with retention times $20.2 \mathrm{~min}, 24.8 \mathrm{~min}, 34.7 \mathrm{~min}$, and 19.0 min, respectively. Because the standard solutions were not further purified, arsenic containing impurities were observed.

Subsequently, aliquots of the arsenite $\mathrm{CH}_{3} \mathrm{~B}_{12}$ methylation mixture, removed after 2 and $3.5 \mathrm{~h}$, were analyzed with this method, and the resulting chromatograms are shown in Figure 5. In Figure 5a, a chromatogram of the nondiluted sample taken after $2 \mathrm{~h}$ incubation at $37^{\circ} \mathrm{C}$ is presented. Because the concentration of the ATG complex is very high and to prevent the ICP-MS detector from damage during the time interval that this compounds elutes from the column (18-22 $\mathrm{min}$ ), the eluent was diverted from the ICP-MS. In this sample, the presence of the MADG complex having a retention time of $24.6 \mathrm{~min}$ is evident.

In the chromatogram shown in Figure $5 b$, the analysis of an aliquot from the reaction mixture (diluted 1:200 with nanopure water) after $3.5 \mathrm{~h}$ incubation is presented. The peak with a retention time of $20.2 \mathrm{~min}$ corresponds to ATG, as it matches that of its corresponding standard.

Because the retention times of the complexes present in standard solutions and the methylation reaction mixture were similar ( $<2 \%$ difference), it was not necessary to conduct spiking experiments to confirm their presence in the analyzed sample. In addition, because of questionable compound stability, spiking may not afford credible results.

Chromatographic recovery for the HPLC-ICP-MS method was determined offline by fraction collection. First, a mixture of the four As-GSH complexes was

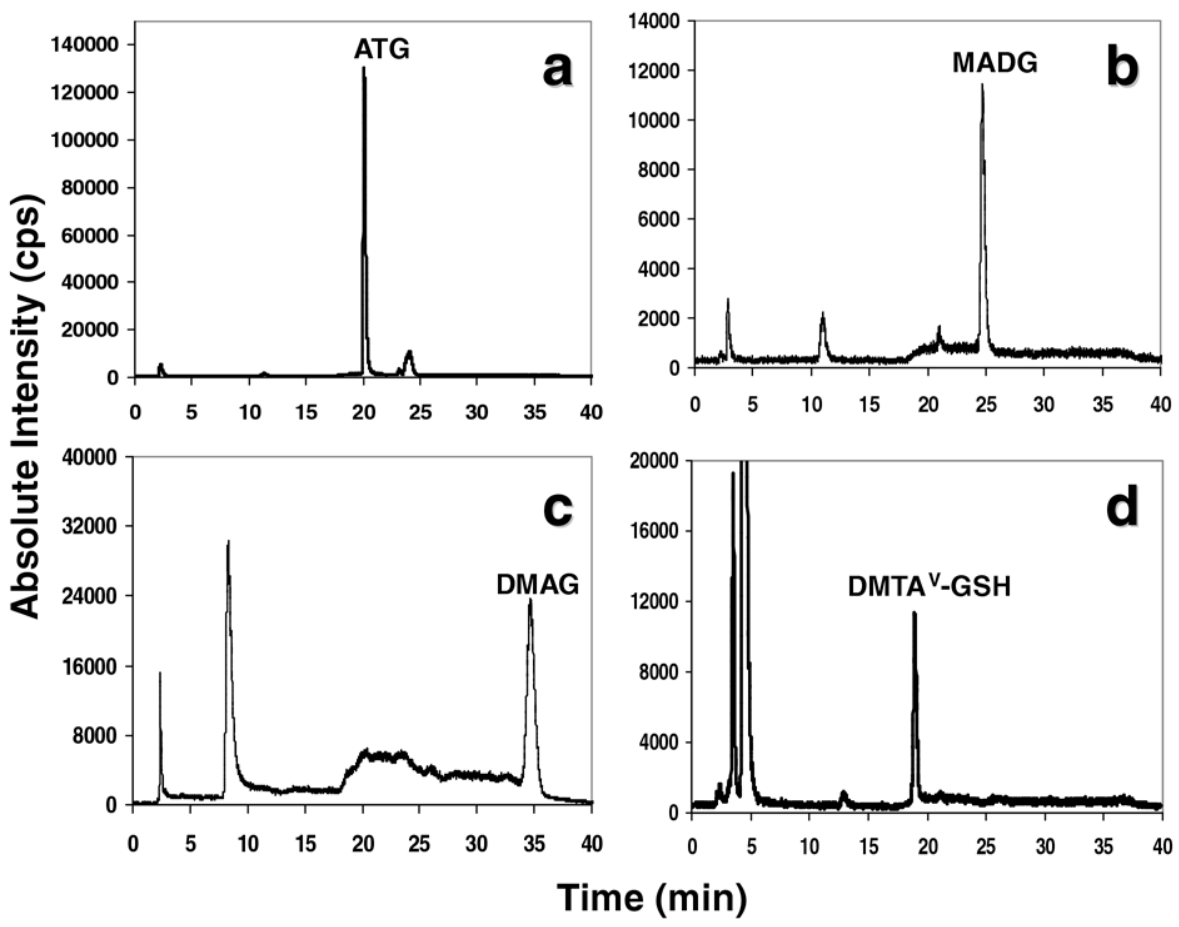

Figure 4. Reversed-phase HPLC-ICP-MS chromatogram of standard solutions of: (a) ATG (3.5 $\mu \mathrm{g} / \mathrm{mL}),(\mathbf{b})$ MADG $(0.58 \mu \mathrm{g} / \mathrm{mL}),(\mathbf{c})$ DMAG $(2.2 \mu \mathrm{g} / \mathrm{mL})$, and (d) DMTA ${ }^{\mathrm{V}}-\mathrm{GSH}(0.55 \mu \mathrm{g} / \mathrm{mL})$. The other observed peaks in each chromatogram correspond to impurities. 
injected onto the HPLC system and the eluent was collected for the entirety of the chromatographic run (Fraction I). Second, injection of the same sample was repeated, but without the HPLC column, and again the analytes were collected (Fraction II). In both cases, blank fractions consisting of mobile phase were collected and used to correct for the arsenic background in Fractions I and II. The arsenic response of all fractions was measured using ICP-MS. The percentage chromatographic recovery for arsenic was calculated as:

$$
\left[\frac{\text { (blank corrected arsenic intensity of fraction I) }}{\text { (blank corrected arsenic intensity of fraction II) }}\right]
$$$$
\times 100
$$

The calculated chromatographic recovery was $83 \%$.

\section{Estimated Limits of Detection for the Four As-GSH Complexes Using HPLC-ICP-MS and HPLC-ES-MS/MS Methods}

To determine the limits of detection (LODs) for the four As-GSH species using both HPLC-ICP-MS and HPLC-

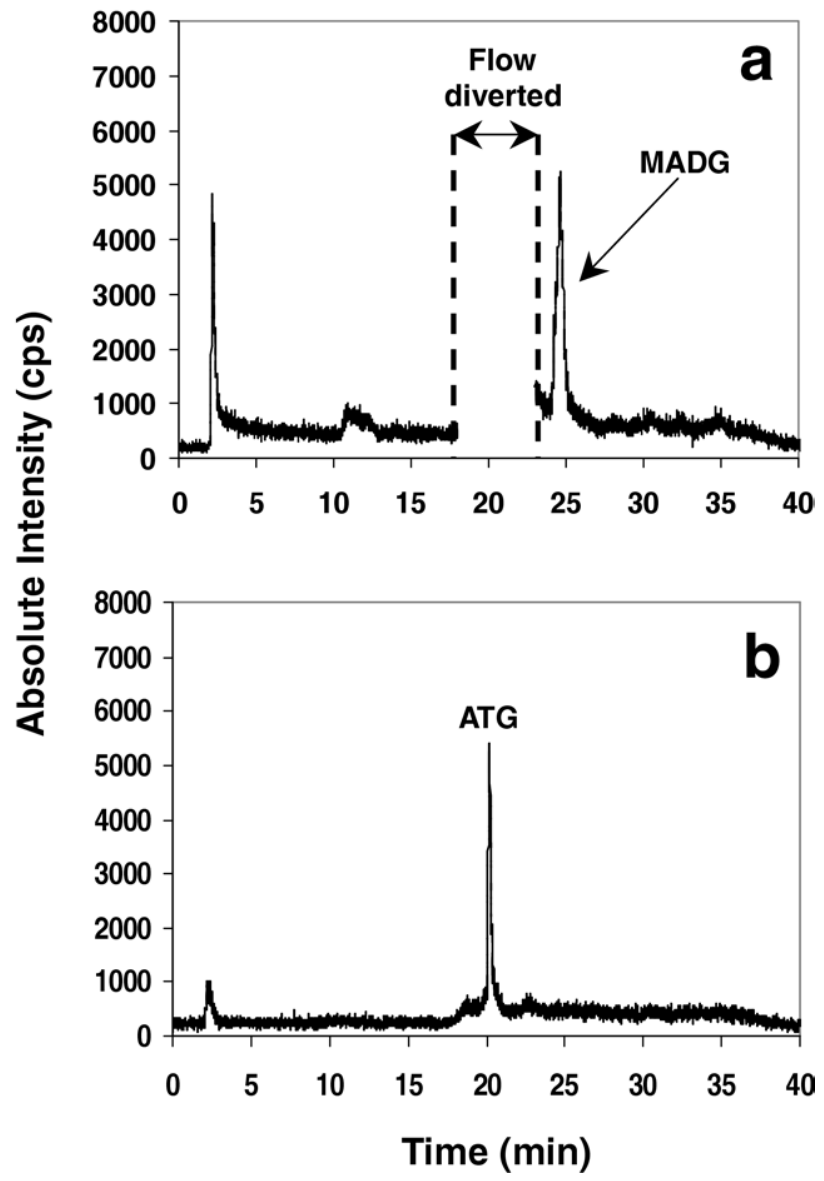

Figure 5. Reversed-phase HPLC-ICP-MS chromatogram of the arsenite methylation reaction mixture: (a) nondiluted aliquot after $2 \mathrm{~h}$ incubation at $37^{\circ} \mathrm{C}$ and (b) diluted sample (1:200 with deionized water) after $3.5 \mathrm{~h}$ incubation at $37^{\circ} \mathrm{C}$.
Table 2. Estimated LODs for the four As-GSH complexes using HPLC-ICP-MS and HPLC-ES-MS/MS-SRM methods

\begin{tabular}{lccc}
\hline & & \multicolumn{2}{c}{ LODs $(\mu \mathrm{g} / \mathrm{L})$} \\
\cline { 3 - 4 } Compound & $\begin{array}{c}\text { \% Vol/vol } \\
\text { methanol } \\
\text { content }^{\mathrm{a}}\end{array}$ & $\begin{array}{c}\text { HPLC-ICP- } \\
\text { MS }\end{array}$ & $\begin{array}{c}\text { HPLC-ES-MS/MS-SRM } \\
\text { (SRM transitions }\end{array}$ \\
\hline \hline ATG & 12 & 56 & 1123 \\
MADG & 12 & 26 & 210 \\
DMAG & 2 & 40 & 8 \\
DMTA $^{\mathrm{V}}$-GSH & 12 & 20 & 0.2 \\
\hline
\end{tabular}

${ }^{a}$ Methanol content of mobile phase during the elution of each arsenic species.

bSRM transitions used for each compound are given in Table 1.

ES-MS/MS-SRM methods, the standard solutions of these species were serial-diluted in $0.1 \%$ formic acid and analyzed. The LOD was considered the lowest analyte concentration that produces a response three times the chromatographic background noise level. The resulting LODs, expressed as $\mu \mathrm{g}$ As/L solution are listed in Table 2.

As observed from Table 2, all four complexes exhibit similar HPLC-ICP-MS LODs. The small differences that are observed may be attributed to the applied gradient program, during which the methanol content in the mobile phase increases from $2 \%$ to $12 \% \mathrm{vol} / \mathrm{vol}$. This increase in the methanol amount causes variations in the ICP-MS sensitivity and background.

With respect to the HPLC-ES-MS/MS-SRM method, the LODs determined for the four As-GSH species differ significantly. This is expected as it is well known that the sensitivity and the LODs of this method are species-dependent. Thus, arsenic compounds with different hydrophobicities, oxidation states, and degree of methylation exhibit sensitivity and LOD variations.

The DMAG complex is detected at lower LODs using HPLC-ES-MS/MS-SRM compared with using HPLCICP-MS, which explains why this compound was detectable as a product of the methylation reaction when using the former technique and not detected using the latter.

\section{Conclusions}

This study has demonstrated the development of mass spectrometric techniques, i.e., HPLC-ES-MS/MS-SRM and HPLC-ICP-MS, for the selective identification of four As-GSH species (ATG, MADG, DMAG, and DMTA $^{\mathrm{V}}-\mathrm{GSH}$ ) in synthetic standard solutions. Furthermore, this methodology was applied to carry out a preliminary study of the nonenzymatic methylation of arsenite by methylcobalamin in the presence of glutathione and for the first time the arsenic complexes ATG, MADG, and DMAG were detected as products of this reaction. These three species are possible intermediates in the arsenic biomethylation process. 


\section{Acknowledgments}

The authors thank the European Commission for funding of a Marie Curie Excellence Grant (contract MEXT-CT-2003-002788).

\section{References}

1. Mandal, B. K.; Suzuki, K. T. Arsenic Round the World: A Review. Talanta 2002, 58, 201-235.

2. Smith, A. H.; Hopenhayn-Rich, C.; Bates, M. N.; Goeden, H. M.; Hertz-Picciotto, I.; Duggan, H. M.; Wood, R.; Kosnett, M. J.; Smith, M. T. Cancer Risks from Arsenic in Drinking Water. Environ. Health Perspect. 1992, 97, 259-267.

3. Suzuki, K. T.; Mandal, B. K.; Katagiri, A.; Sakuma, Y.; Kawakami, A.; Ogra, Y.; Yamaguchi, K.; Sei, Y.; Yamanaka, K.; Anzai, K.; Ohmichi, M.; Takayama, H.; Aimi, N. Dimethylthioarsenicals as Arsenic Metabolites and Their Chemical Preparations. Chem. Res. Toxicol. 2004, 17, 914-921.

4. Le, X. C.; Lu, X.; Ma, M.; Cullen, W. R.; Aposhian, H. V.; Zheng, B. Speciation of Key Arsenic Metabolic Intermediates in Human Urine. Anal. Chem. 2000, 72, 5172-5177.

5. Challenger, F. Biological methylation. Chem. Rev. 1945, 36, 315-361.

6. Hayakawa, T.; Kobayashi, Y.; Cui, X.; Hirano, S. A. New Metabolic Pathway of Arsenite: Arsenic-Glutathione Complexes are Substrates for Human Arsenic Methyltransferase Cyt19. Arch. Toxicol. 2005, 79, 183191.

7. Hansen, H. R.; Pickford, R.; Thomas-Oates, J.; Jaspars, M.; Feldmann, J. 2-Dimethylarsinothioyl Acetic Acid Identified in a Biological Sample: The First Occurrence of a Mammalian Arsinothioyl Metabolite. Angew. Chem. Int. Ed. 2004, 116, 341-344.

8. Schmeisser, E.; Raml, R.; Francesconi, K. A.; Kuehnelt, D.; Lindberg, A.-L.; Sörös, C.; Goessler, W. Thio Arsenosugars Identified as Natural Constituents of Mussels by Liquid Chromatography Mass Spectrometry. Chem. Commun. 2004, 16, 1824-1825.

9. Fricke, M. W. Creed, P. A. Parks, A. N. Shoemaker, J. A. Schwegel, C. A.; Creed, J. T. Extraction and Detection of a New Arsine Sulfide Containing Arsenosugar in Mollusks by IC-ICP-MS and IC-ESI-MS/ MS. J. Anal. At. Spectrom. 2004, 19, 1454-1459.

10. Nischwitz, V.; Kanaki, K.; Pergantis, S. A. Mass Spectrometric Identification of Novel Arsinothioyl-sugars in Marine Bivalves and Algae. I. Anal. At. Spectrom. 2006, 21, 33-40.

11. Raab, A.; Schat, H.; Meharg, A. A.; Feldmann, J. Uptake, Translocation and Transformation of Arsenate and Arsenite in Sunflower (Helianthus annuus): Formation of Arsenic-Phytochelatin Complexes During Exposure to High Arsenic Concentrations. New Phytologist 2005, 168, 551-558.
12. Kala, S. V.; Neely, M. W.; Kala, G.; Prater, C. I.; Atwood, D. W.; Rice, J. S.; Lieberman, M. W. The MRP2/cMOAT Transporter and ArsenicGlutathione Complex Formation are Required for Biliary Excretion of Arsenic. J. Biol. Chem. 2000, 275, 33404-33408.

13. Kobayashi, Y.; Cui, X.; Hirano, S. Stability of Arsenic Metabolites, Arsenic Triglutathione $[A s(G S) 3]$ and Methylarsenic Diglutathione [CH3As(GS)2] in Rat Bile. Toxicology 2005, 211, 115-123.

14. Cui, X.; Kobayashi, Y.; Hayakawa, T.; Hirano, S. Arsenic Speciation in Bile and Urine Following Oral and Intravenous Exposure to Inorganic and Organic Arsenics in Rats. Toxicol. Sci. 2004, 82, 478-487.

15. Kala, S. V.; Kala, G.; Prater, C. I.; Sartorelli, A. C.; Lieberman, M. W. Formation and Urinary Excretion of Arsenic Triglutathione and Methylarsenic Diglutathione. Chem. Res. Toxicol. 2004, 17, 243-249.

16. Raab, A.; Wright, S. H.; Jaspars, M.; Meharg, A. A.; Feldmann, J. Pentavalent Arsenic Can Bind to Biomolecules. Angew. Chem. Int. Ed. 2007, 46, 2594-2597.

17. Raab, A.: Meharg, A. A.; Jaspars, M.; Genneyb, D. R.; Feldmann, J Arsenic-Glutathione Complexes-Their Stability in Solution and During Separation by Different HPLC Modes. J. Anal. At. Spectrom. 2004, 19, $183-190$.

18. Scott, N.; Hatlelid, K. M.; MacKenzie, N. E.; Carter, D. E. Reactions of Arsenic(III) and Arsenic(V) Species with Glutathione. Chem. Res. Toxicol. 1993, 6, 102-106.

19. Delnomdedieu, M.; Basti, M. M.; Otvos, J. D.; Thomas, D. J. Reduction and Binding of Arsenate and Dimethylarsinate by Glutathione: A Magnetic Resonance Study. Chem. Biol. Interact. 1994, 90, 139-155.

20. Styblo, M.; Yamauchi, H.; Thomas, D. Comparative in Vitro Methylation of Trivalent and Pentavalent Arsenicals. Toxicol. Appl. Pharmacol. 1995, 135, 172-178

21. Styblo, M.; Thomas, D. J. In Vitro Inhibition of Glutathione Reductase by Arsenotriglutathione. Biochem. Pharmacol. 1995, 49, 971-977.

22. Burford, N.; Eelman, M. D.; Groom, K. Identification of Complexes Containing Glutathione with $\mathrm{As}(\mathrm{III}), \mathrm{Sb}(\mathrm{III}), \mathrm{Cd}(\mathrm{II}), \mathrm{Hg}(\mathrm{II}), \mathrm{Tl}(\mathrm{I}), \mathrm{Pb}(\mathrm{II})$, or $\mathrm{Bi}(\mathrm{III})$ by Electrospray Ionization Mass Spectrometry. Inorg. Biochem. 2005, 99, 1992-1997.

23. Zakharyan, R. A.; Aposhian, H. V. Arsenite Methylation by Methylvitamin B12 and Glutathione Does Not Require an Enzyme. Toxicol. Appl. Pharmacol. 1999, 154, 287-291.

24. Pergantis, S. A.; Miguens-Rodriguez, M.; Vela, N. P.; Heitkemper, D. T. Investigating the Nonenzymatic Methylation of Arsenite by Methylcobalamin B12 using High-Performance Liquid Chromatography On-Line with Inductively Coupled Plasma-Mass Spectrometry. J. Anal. At. Spectrom. 2004, 19, 178-182.

25. Nischwitz, V.; Pergantis, S. A. Liquid Chromatography On-Line with Selected Reaction Monitoring Electrospray Mass Spectrometry for the Determination of Organoarsenic Species in Crude Extracts of Marine Reference Materials. Anal. Chem. 2005, 77, 5551-5563. 